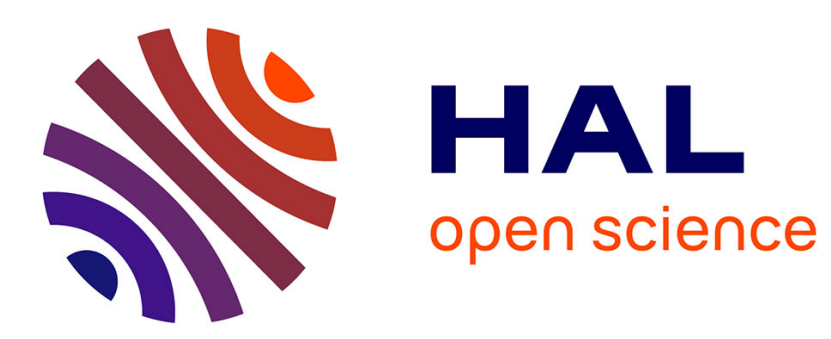

\title{
Évolution épistémologique en EIAO
}

Pierre Dillenbourg

\section{To cite this version:}

Pierre Dillenbourg. Évolution épistémologique en EIAO. Sciences et Techniques Educatives, 1994, 1 (1), pp.39-51. edutice-00001442

HAL Id: edutice-00001442

https://edutice.archives-ouvertes.fr/edutice-00001442

Submitted on 5 Apr 2006

HAL is a multi-disciplinary open access archive for the deposit and dissemination of scientific research documents, whether they are published or not. The documents may come from teaching and research institutions in France or abroad, or from public or private research centers.
L'archive ouverte pluridisciplinaire HAL, est destinée au dépôt et à la diffusion de documents scientifiques de niveau recherche, publiés ou non, émanant des établissements d'enseignement et de recherche français ou étrangers, des laboratoires publics ou privés. 


\section{Évolution épistémologique en EIAO}

\section{Pierre Dillenbourg}

TECFA Faculté de Psychologie et des Sciences de l'Éducation

Université de Genève 9, Route de Drize, CH1227 Carouge (Suisse)

Tél. 022/705.96.93 Fax. 022/342.89.24

Courrier électronique: pdillen@divsun.unige.ch

RESUME. Le concept de connaissance a fortement évol ué au cours des dernières années. La connaissance n'est plus perçue comme une substance, mais comme une capacité à interagir. Jillustre cette évolution épistémologique par deux exemples: l'explication et la modélisation de l'apprenant. Initialement considérée comme la transmission d'une trace du raisonnement de l'expert, l'explication est aujourd'hui vue comme le résultat d'une construction commune. De façon similaire, le diagnostic n'est plus considéré comme une photographie neutre des connaissances du sujet, mais comme le résultat d'un processus interactif de compréhension mutuelle.

ABSTRACT. The concept of knowledge has recently evolved from a substance to a capacity to interact. I illustrate this epistemological evolution with two examples: explanation and learner modelling. Explanation is not perceived any more as transmiting the trace of the expert's reasoning, but as the result of a shared construction. Similarily, a diagnosis is not considered any more as a neutral snapshot of the learner's knowledge, but as the result of a mutual understanding process.

MOTS-CLES : cognition située, cognition distribuée, connaissance, explication, modélisation de l'apprenant.

KEYWORDS : situated cognition, distributed cognition, knowledge, explanation, learner modelling.

Cet article concerne l'évolution des travaux sur les applications de l'intelligence artificielle à l'éducation. Ces travaux sont généralement désignés par le sigle EIAO (environnements interactifs d'apprentissage 
avec ordinateur). Au cours des cinq dernières années, les bases conceptuelles de l'EIAO ont été remises en cause par des critiques d'ordre technique ou théorique. Les premières résultent des difficultés qu'ont rencontrées les concepteurs d'EIAO. Les secondes, plus théoriques, s'adressent à l'approche cognitive sur laquelle l'EIAO repose. Ces critiques sont émises par les promoteurs du courant 'situated cognition', dont je désignerai les défenseurs par le terme "contextualistes". Cet article ne propose ni une discussion des fondements théoriques des thèses contextualistes (fonctionnement de la mémoire, dimension sociale des processus cognitifs, ...), ni une prédiction de l'impact de ces théories sur l'évolution de l'EIAO [CLA 93, DIS 93]. Il se centre sur l'évolution du concept de connaissances au sein des travaux d'EIAO.

Les thèses contextualistes n'ont pas (encore) convaincu la majorité des chercheurs en intelligence artificielle (IA) et en EIAO, surtout en Europe. Cependant, on peut clairement noter une évolution du conceptclé de l'EIAO: la connaissance. Pendant deux décennies, la connaissance a été considérée comme une "substance mentale" [SAN 82, CLA 92], dotée de propriétés presque matérielles. Ces propriétés permettent notamment de représenter, de manipuler et de communiquer cette connaissance. Le concept de connaissance-substance a traditionnellement été associé aux représentations symboliques de cette connaissance. Cette image n'a pas totalement disparu, mais ses propriétés dynamiques ont été révélées, en particulier en mettant en évidence les relations entre les connaissances et le contexte physique et social dans lequel ces connaissances sont mises en œuvre. Aujourd'hui, les contextualistes redéfinissent la connaissance comme une capacité à interagir. Cette nouvelle image n'est pas encore très précise. Aussi, je tenterai de la concrétiser en décrivant l'évolution de deux problématiques présentes en EIAO : l'explication et la modélisation de l'apprenant.

\section{Bref historique: évolution du concept de connaissance}

Pour comprendre l'évolution actuelle des recherches en EIAO, il n'est pas inutile de dresser un bref historique de cette discipline. Son histoire commence en 1970, lorsque Carbonell [CAR70] publie ses travaux sur SCHOLAR, qui est généralement considéré comme la premier système de cette discipline. Durant une décennie, la communauté EIAO reste restreinte et confidentielle. Elle n'en jette pas moins les bases conceptuelles qui constituent encore aujourd'hui le vocabulaire de base de l'EIAO : le modèle de l'élève, le modèle du domaine et le modèle pédagogique. En 1982, Sleeman et Brown publient le premier ouvrage 
consacré spécifiquement à l'EIAO [SLE 82]. Sa parution conduira à un élargissement rapide de la communauté. Les années quatre-vingt ne remettent pas en cause les fondements théoriques élaborés au cours de la décennie précédente. Néanmoins, l'intensité croissante des recherches permet de tester plus intensivement les postulats de base, de prendre conscience d'autres postulats demeurés implicites et de mettre en évidence certaines limites théoriques et techniques des modèles en vigueur. Cette première période est admirablement résumée dans l'ouvrage de Wenger [WEN 87]. Dès la fin des années 80, plusieurs voix remettent en cause les fondements de l'intelligence artificielle et l'approche cognitive en psychologie [WIN 86, SUC 87, LAV 88, BRO 91]. Ces critiques atteignent rapidement la communauté EIAO [BRO 90]. Elles marquent le début d'une seconde période, qui se différencie de la première par l'idée que se font les chercheurs du concept de connaissance.

Dès le début, le concept de connaissance fut au coeur des travaux en EIAO. En effet, cette discipline a acquis son identité en prenant ses distances vis-à-vis des théories comportementales qui dominaient alors la scène de l'EAO. Dans la perspective 'béhavioriste', l'apprentissage était considéré comme le résultat du renforcement systématique de comportements adéquats. Plus tard, lorsque la psychologie cognitive prend le pas sur le béhaviorisme, apprendre devient synonyme d'acquérir les connaissances nécessaires à la mise en œuvre du comportement-cible. L'EIAO ne s'écarte pas uniquement de l'approche comportementale, mais également des premiers travaux de l'intelligence artificielle. Au mythe de la résolution générale de problèmes [NEW 72], l'EIAO préfère la puissance des connaissances spécifiques, puissance illustrée par les succès contemporains des concepteurs de systèmes experts.

La possibilité de stoker et d'exécuter les connaissances du domaine d'enseignement constitue la clef de voûte des tutoriels intelligents. La représentation de ces connaissances permet de ne pas traiter uniquement les réponses, mais de pénétrer dans le processus même de résolution du problème. Ces connaissances sont stockées dans ce qu'on appelle le modèle du domaine ou le modèle de l'expert. Ce noyau nourrit directement ses deux appendices, le modèle de l'élève et le modèle pédagogique. Le modèle de l'élève se définit le plus souvent comme une perturbation du modèle de l'expert (par omission, adjonction ou substitution de connaissances) telle que le modèle perturbé génère un comportement semblable à celui de l'élève. Le rôle du modèle pédagogique - dans les rares cas où celui-ci fut réellement développé - se limite généralement à la sélection des connaissances de l'expert sur lesquelles portera la prochaine intervention du système (construction 
dynamique du curriculum). Dans les années 80 , la prépondérance des connaissances du domaine est telle qu'on décrit l'architecture d'un tutoriel par la trilogie "expert - tuteur - élève", c'est-à-dire en oubliant le rôle essentiel de l'interface apprenant-système.

Comme l'indique Wenger dans le titre même de son ouvrage [WEN 87], ces systèmes sont essentiellement perçus comme des dispositifs de communication des connaissances. Contrairement à certaines critiques, l'utilisation du terme "communication" ne témoigne pas d'une conception pédagogique selon laquelle il suffirait de présenter certaines connaissances à l'élève pour que celui-ci les assimile (conception dite des "vases communiquants"). En réalité, dès le départ, la communauté EIAO s'inscrit principalement dans une perspective constructiviste (malgré quelques exceptions notables). Par contre, cette communauté partage une certaine conception épistémologique. La connaissance semble être perçue comme une substance, dotée de propriétés presque matérielles. Ce biais matérialiste sera décrit plus facilement en montrant comment certains chercheurs s'en sont écartés.

Un des facteurs de l'évolution que je tente de décrire réside dans les problèmes complexes de représentation des connaissances auxquels ont été confrontés les chercheurs en IA et en EIAO. Il est progressivement apparu que le choix d'un mode de représentation n'est pas indépendant de l'utilisation de ces connaissances au sein d'un tutoriel. Dans les simulations par exemple, le modèle du domaine est soumis à des exigences partiellement incompatibles de performance (produire une réponse correcte et rapide) et de transparence (pourvoir expliquer comment les résultats ont été obtenus) [WEN 87]. Face à cette incompatibilité, les auteurs suggèrent d'utiliser plusieurs représentations du domaine car:

- des représentations multiples permettent de couvrir des fonctions pédagogiques multiples telles que le diagnostic, l'explication et la simulation [ROS 88];

- des modèles hiérarchisés permettent d'adapter les interactions aux différents stades du développement de l'apprenant [FRE 88];

- des modèles équivalents permettent au système d'interagir sur le domaine selon différents points de vue et donc de s'adapter au point de vue du sujet [MOY 92]. 
Le besoin de connaissances multiples pourrait être considéré comme un problème technique ne remettant pas en cause l'unicité de l'objet représenté. Cependant, avec le recul, on peut se demander si ces différentes représentations ne correspondent pas en réalité à des connaissances différentes! Dans la perspective contextualiste, il s'agirait en fait de plusieurs capacités à interagir, qui partagent certes un objet commun, mais n'en sont pas moins différenciables. Sans répondre définitivement à cette question, on peut constater que le besoin de représentations multiples constitue un premier signe de l'affaiblissement du concept de "connaissance-substance".

\section{Evolution des travaux sur l'explication}

L'explication a initialement été conçue comme un effet de bord de la résolution de problème. Expliquer consiste à mettre en forme la trace du raisonnement de l'expert. Techniquement, cela consiste à générer un 'texte' à partir de la liste des règles utilisées et à adapter ce texte aux interrogations et au niveau de compréhension du sujet. La forme d'adaptation la plus fréquente consiste à jouer avec la granularité de l'explication. La granularité est maximale lorsque chaque règle utilisée par l'expert figure dans l'explication. Cette forme d'adaptation correspond à une problématique très empreinte de paternalisme pédagogique : faut-il tout expliquer? Cette approche témoigne d'une conception de l'explication comme un objet que l'on peut remettre à quelqu'un, comme une information transmise de A vers B.

Le premier signe d'une évolution apparaît lorsque certains auteurs transforment cette explication statique en objet interactif : la trace est produite sous la forme d'un hypertexte, l'apprenant peut choisir lui-même le niveau de granularité de l'explication qui lui convient. Un autre indice d'évolution figure dans les travaux de Vivet [VIV 88]. Leur système AMALIA utilise des métarègles pour guider le raisonnement de l'expert sur les chemins que l'élève peut comprendre (par exemple en évitant d'utiliser des règles que l'élève ne maitrise pas). Certes, la résolution du problème et l'explication demeurent deux étapes bien distinctes dans le temps, mais elles ne sont plus totalement indépendantes: la fonction 'explication' influence le raisonnement. Progressivement, le caractère interactif de l'explication s'affirme, notamment sous la forme de sousdialogues de clarification. Ces sous-dialogues peuvent encore être interprétés à l'intérieur du paradigme en vigueur, comme des outils permettant de régler localement le niveau de granularité ou de préciser un terme qui n'a pas été compris. Néanmoins, au terme de 
l'expérimentation du système ELISE, issu des travaux sur CAMALIA, Delozanne conclut que "c'est bien l'ensemble de l'interaction qui est explicative, et non pas le seul contenu des messages explicatifs ponctuels." [DEL 93]

La profonde interdépendance entre l'explication et le raisonnement est mise en évidence - ce n'est pas étonnant - par les psychologues qui tentent de comprendre les bénéfices de l'auto-explication [CHI 89]. Comment comprendre qu'un individu améliore ses connaissances en s'expliquant quelque chose qu'il connaît déjà (puisqu'il est capable de se l'expliquer)? Les réponses à cette question sont liées à la procéduralisation des connaissances : expliquer, c'est transformer des connaissances déclaratives et générales en connaissances opérationnelles et contextualisées. Ces recherches montrent que l'explication n'est pas un simple effet de bord d'un raisonnement, mais qu'elle constitue un raisonnement à part entière. En dehors de certaines explications fossilisées à force de répétition ("mais comment t'es-tu cassé le pied?"), l'explication résulte d'une construction. Le processus explicatif ne consiste pas à construire puis transmettre une structure plus ou moins figée. Il s'agit d'un processus plus complexe par lequel deux personnes essaient de construire ensemble une nouveau schéma permettant de comprendre ou de résoudre un problème [OMA 87, BAK 92, CAW 93]. Cette conception de l'explication est plus facile à saisir lorsqu'on parle de la collaboration entre deux sujets de niveaux équivalents. En effet, les travaux récents sur l'apprentissage collaboratif considère celui-ci comme la co-construction d'une représentation du problème et de sa solution [ROS 92]. L'élaboration d'un schéma commun requiert une négociation au cours de laquelle chaque élément peut être discuté, précisé, généralisé ou réfuté, de façon récursive ${ }^{1}$ jusqu'au moment où il est mutuellement accepté [BAK 93].

On peut résumer cette évolution de la manière suivante. Construire une explication n'est plus perçu comme ouvrir une fenêtre sur un processus afin d'en permettre une observation neutre. Parce que l'explication est fondamentalement interactive et qu'elle interfère directement avec le processus de résolution de problème, elle transforme ce dernier en processus interactif.

\section{Evolution des travaux sur la modélisation de l'apprenant}

1 Par exemple, la réfutation d'un réfutation. 
Les travaux sur la modélisation de l'apprenant ont suivi une évolution semblable à celle des recherches sur l'explication. Ce parallélisme n'est pas surprenant car l'explication et la modélisation peuvent être considérées comme des processus réciproques. Au fur et à mesure que nous pénétrerons dans la dimension interactive de ces processus, ceux-ci apparaîtront comme deux façons de considérer un même objet scientifique.

La modélisation de l'apprenant constitue une problématique de l'EIAO au sein de laquelle les sciences cognitives, en particulier le concept de représentation mentale, ont laissé de profondes empreintes. La conception initiale de ces représentations est presque matérielle. Cette matérialité ne concerne pas le substrat physiologique des représentations, mais leur communicabilité et la possibilité de les manipuler. Comme pour l'explication, les rôles étaient initialement clairement différenciés: l'apprenant était l'objet modélisé, le tutoriel réalisait le diagnostic. Cependant, la mise en œuvre d'un diagnostic fondé sur cette approche a, au fil des années, révélé un certain nombre de problèmes difficiles, voire insolubles. Ces difficultés ont poussé les chercheurs à explorer de nombreuses alternatives [DIS 92a, DIS 92b] qui vont progressivement affaiblir la position épistémologique "forte" du départ. Face à ces difficultés, Self suggère certaines solutions [SEL 88] que je vais discuter ici: l'utilisation de représentations intermédiaires, l'utilisation de systèmes de croyances et la prise en compte des possibilités d'exploitation du diagnostic.

La première suggestion consiste à faire participer l'apprenant au diagnostic en lui demandant de formuler des représentations intermédiaires. Twidale [TWI 89] applique ces principes au logiciel EPIC dans lequel il demande aux sujets d'exprimer leurs plans au cours de la démonstration d'un théorème de logique. Confirmant les affirmations de Suchman [SUC 87], les sujets de Twidale déclarent qu'ils n'avaient pas de plan a priori mais que, contraints à en formuler, ils ont finalement trouvé cet artefact très utile. En d'autres termes, le diagnostic perd sa neutralité dès qu'il devient interactif. Cet énoncé a les saveurs d'une tautologie puisque le terme interactif désigne précisément l'interpénétration de plusieurs processus. Il n'est cependant pas évident que ce constat soit explicite pour l'ensemble des chercheurs du domaine. Il constitue pourtant un principe de base de l'école socioculturelle, mouvement qui trouve ses origines dans les années 30, mais qui connaît une nouvelle jeunesse, notamment depuis la traduction des ouvrages de Vygotsky [VYG 78]. La relation entre les effets de bord du diagnostic et l'approche socioculturelle peut se résumer par le slogan "participation changes understanding" [ROG 91] : la perception de la façon dont une 
autre personne a compris nos actions modifie notre propre compréhension de nos actions [NEW 89]. Ce mécanisme, connu sous le nom d'appropriation, est le pendant social du concept d'assimilation que Piaget emprunta à la biologie [NEG 89].

Si le diagnostic influence les connaissances de l'élève, cela pose un problème quant à la cohérence de diagnostics successifs. Imaginons qu'une erreur se produise au cours d'un problème $\mathrm{P} 1$, mais ne soit pas reproduite ensuite pour un problème $\mathrm{P} 2$, considéré comme équivalent à P1. Cette erreur est généralement attribuée à ce qu'on appelle une "cause non-systématique" : fatigue, stress, distraction,...2 La disparition d'une erreur peut également être liée à un mécanisme d'apprentissage, par exemple dû au premier diagnostic (dans P1). Une autre possibilité peut être envisagée: si les problèmes $\mathrm{P} 1$ et $\mathrm{P} 2$ sont considérés comme équivalents par un expert, ils peuvent par contre paraître comme des problèmes différents aux yeux de l'apprenant. En effet, les novices ne perçoivent pas nécessairement les situations selon les mêmes critères que les experts. Il arrive que l'apprenant mette en œuvre des connaissances que nous jugeons contradictoires dans des contextes pourtant similaires, parce qu'il perçoit ces contextes comme différents. La relativité des connaissances par rapport au contexte de leur acquisition ou de leur utilisation constitue la clef de voûte des arguments défendus par les contextualistes. Certaines techniques de représentation des connaissances, appelées systèmes de croyances ("beliefs systems") permettent d'exprimer certaines formes de cette relativité:

- relativité au contexte : "croit [neige en décembre, France]; croit [pas de neige en décembre, Espagne]";

- relativité au possesseur de la connaissance : "croit [Jacques, neige en décembre, France]";

- degré de conviction : "croit [Paul, baisse du chômage, France, $0.45]^{\prime \prime}$ signifie que Paul ne croit pas vraiment qu'il y aura une baisse du chômage en France.

Le concept de croyance constitue un signe de l'évolution épistémologique de la discipline dans la mesure où il correspond à une vue moins statique que le concept original de connaissances. En outre, ce

2 On parle par contre de cause systématique lorsque l'erreur est due à un défaut dans les connaissances du sujet. 
concept permet de représenter le diagnostic en tant que croyance du système sur les croyances du sujet: "[croit [system, croit [Paul; baisse du chômage, France, 0.45], début, 0. 86] " signifie que le système est assez sûr $(86 \%)$ que Paul ne croit pas vraiment qu'il y aura une baisse du chômage en France.

Outre l'élaboration du diagnostic, les chercheurs se sont également souciés de l'utilisation du diagnostic par le système. Si le système doit choisir entre $\mathrm{N}$ stratégies, il n'a besoin que de discriminer $\mathrm{N}$ profils différents [OHL 87; SEL 88; DIL 89]. Tout diagnostic plus fin que ces $\mathrm{N}$ catégories constitue une performance peut-être remarquable, mais probablement inutile. La prise en compte du rôle du diagnostic réunit progressivement deux processus, diagnostiquer et intervenir, qui avaient été artificiellement séparés par les architectures des systèmes d'EIAO. Cette évolution amène Self à proposer de rendre le modèle de l'élève inspectable [SEL 88]. ${ }^{3}$ Bull, Pain et Brna [BUL 93] franchissent un pas de plus dans cette évolution en permettant au sujet de rectifier les éventuelles erreurs de diagnostic (de son point de vue). Un modèle inspectable et modifiable fournit au sujet un feed-back sur ses connaissances et au système un feed-back sur la qualité de son diagnostic.

Cette évolution aboutit à une conception du diagnostic comme un processus collaboratif et mutuel. Dans les études sur le dialogue, ce mécanisme est en fait connu sous le nom d'ancrage social ("social grounding")[CLA 91]. Il désigne les mécanismes par lesquels une personne s'assure que son interlocuteur a compris ce qu'elle disait, probablement pas parfaitement, mais pour le moins, à un niveau permettant de continuer l'interaction. Dans les conversations entre personnes, l'émetteur dispose de nombreux indices pour surveiller la compréhension du récepteur : froncement de sourcils, interruption, perte d'attention,... Il dispose également de mécanismes pour réparer les problèmes de communication, notamment pointer physiquement vers des objets ou faire référence à des faits supposés connus par le récepteur. Malheureusement, la communication personne-machine manque encore singulièrement de moyens pour percevoir et réparer les ambiguités de la communication [SUC 87]. Les expériences réalisées avec l'environnement d'apprentissage MEMOLAB [DIH 93] nous fournissent certaines évidences de la réciprocité du processus de diagnostic :

3 Le lecteur attentif percevra ici une fois de plus le parallélisme avec l'évolution des travaux sur l'explication, notamment lorsque certains chercheurs ont suggéré de mettre l'explication à la dispositon de l'apprenant sous la forme d'un hypertexte. 
l'apprenant se forge une idée de ce que la machine sait, et en particulier de ce que la machine sait à son propos. Il se construit donc un diagnostic intuitif du diagnostic élaboré par la machine. Dans la conversation courante, ce méta-diagnostic appartient à la panoplie des techniques naturelles qui permettent de réparer les ambiguités de la communication. En voici un exemple fictif :

Sujet A: "Les nombres se terminant par 2 ou par 4 sont pairs."

Sujet B: "158 est aussi un nombre pair"

Sujet A: "Je n'ai pas dit que seuls les nombres se terminant par 2 et 4 sont pairs, il y a aussi ceux qui se terminent par 0,6 ou 8 "

Le sujet $\mathrm{B}$ surinterprète la première phrase du sujet A. Celui-ci perçoit cette surinterpétation car $B$ produit un contre-exemple qui réfute une généralité que $\mathrm{A}$ n'a pas émise. Le sujet $\mathrm{A}$ reformule donc sa première phrase afin de rectifier cette surinterprétation. Cette reformulation n'est cependant pas pégagogiquement neutre puisqu'elle force $\mathrm{A}$ à reconsidérer la portée exacte de ce qu'il avait dit. Sous les apparences d'une reformulation, A dit en réalité quelque chose de différent de sa première phrase. Qu'il s'agisse de l'explicitation d'éléments connus, mais implicites, ou de la découverte d'éléments nouveaux grâce à la réaction de l'interlocuteur, cette reformulation témoigne dans le deux cas d'un processus d'apprentissage.

L'évolution de ces travaux peut se résumer de la façon suivante: si le diagnostic est une activité mutuelle et réciproque et, s'il est interactif, il ne correspond plus à une observation neutre des connaissances de l'apprenant, mais il acquiert le statut d'une intervention pédagogique. 


\section{Synthèse}

Il existe une double similitude entre l'évolution des travaux sur l'explication et l'évolution des travaux sur la modélisation. Premièrement, deux procédés considérés comme disjoints sont fusionnés. Dans les deux cas, le processus le plus interactif pénètre dans le moins interactif des deux: "expliquer" s'intègre dans "raisonner", "intervenir" s'intègre dans "diagnostiquer". Deuxièmement, la stricte distribution des rôles ("l'expliqueur" contre "l'expliqué" ou le "diagnostiqueur" contre le "diagnostiqué") s'efface progressivement. L'explication et la modélisation apparaissent comme des processus que deux personnes réalisent conjointement et réciproquement. Ces convergences n'ont rien de bien surprenant, l'explication et le diagnostic, lorsqu'ils sont interactifs, constituent deux manières de décrire une même réalité, une même classe d'interactions.

$\mathrm{Au}$ cours de cette évolution, chacun de ces concepts a perdu ses connotations didactiques et se rapproche d'avantage des concepts utilisés pour décrire n'importe quel type de dialogue. Cela n'implique pas que tout dialogue soit pourvu de propriétés pédagogiques intrinsèques ou que toute interaction pédagogique doive ressembler à une banale discussion, mais plutôt que les concepteurs de systèmes interactifs devraient s'inspirer davantage des mécanismes par lesquels deux personnes construisent une compréhension partagée d'un phénomène. Ces mécanismes doivent être transposés dans le registre des interactions disponibles entre une personne et l'ordinateur.

Le choix de ces deux exemples, l'explication et le diagnostic, n'est pas aléatoire. Il s'agit de deux problématiques pour lesquelles l'évolution est particulièrement sensible. D'autres exemples sont cependant disponibles. Je citerais notamment le concept de fidélité épistémique proposé par Wenger [WEN 87] pour décrire la relation sémantique entre les connaissances internes du système et leur représentation à l'écran. Cette définition illustre bien le concept de connaissance-substance. Roschelle [ROS 90] s'est inspiré de ce principe lors de la conception de l'environnement "Envisionning Machine". Il a tenté d'implémenter des représentations épistémiquement fidèles de concepts tels que la vélocité ou l'accélération. Au terme de plusieurs cycles conceptionexpérimentation, il a observé que ces représentations graphiques ne possédaient pas de valeur sémantique absolue, mais qu'elles jouaient néanmoins un rôle essentiel : elles servaient de ressource commune pour supporter le processus d'ancrage social entre deux apprenants. Dans cet 
exemple, comme dans ceux sur l'explication et la modélisation, la connaissance perd son statut de matière relativement inerte, que l'on peut stoker et communiquer. Elle acquiert une dimension dynamique que l'on ne peut décrire qu'en termes d'interaction.

$\mathrm{Au}$ cours de cette évolution, le concept de connaissances a acquis une dimension sociale. Que les origines de certaines connaissances, notamment celles à forte composante verbale, soient de nature sociale n'est pas une idée neuve. L'idée intéressante dans l'approche "cognition distribuée" [RES 91, SHR 92], est l'extension de la dimension sociale au fonctionnement de ces connaissances en situation interactive. Cette vision s'écarte nettement de l'individualisme chronique des sciences cognitives. Elle bénéficie du boom de la télématique qui réintroduit en force une relation sociale dans la micro-informatique. Certes, les implications précises de cette évolution en termes de spécifications de systèmes d'EIAO restent à définir. Le but de cet article était de montrer que cette évolution épistémologique a déjà influencé la conception des systèmes d'EIAO.

\section{Remerciements}

Merci à Nicolas Balacheff, Patrick Mendelsohn et aux relecteurs de cette revue pour leurs précieux commentaires.

\section{Références}

[BAK 92] BAKER M. The collaborative construction of explanations. Paper presented at the "2èmes journées Explication du PRC-GDR-IA du CNRS, Sophia-Antipolis 17-19 juin 1992.

[BAK 93] BAKER M. Negotiation in Collaborative Problem-Solving Dialogues. Rapport CR-2/93. CNRS, Laboratoire IRPEACS, Equipe Coast, Ecole Normale Supérieure de Lyon. 1992

[BRO 90] BROWN J.S. Toward a new epistemology for learning. In C. Frasson \& G. Gauthier (Eds). Intelligent tutoring systems at the Crossroad of AI \& Education. Norwood, NJ; Ablex. 1990

[BRO 91] BROOKS R. Intelligence without representation. Artificial intelligence, 47, 139-159, 1991.

[BUL 93] BULL S., PAIN H. \& BRNA P. Collaboration and Reflection in the Construction of a Student Model for Intelligent Computer Assisted Language Learning. Proceedings of the Seventh International PEG Conference, Edinburgh [UK], July 1993. 
[CAR 70] CARBONELL J.R. AI in CAI: an artificial intelligence approach to computer-assiste instruction. IEEE Transactions on Man-Machine Systems, vol. 11, n 4, 1970, pp. 190-202.

[CAW 93] CAWSEY A. Planning Interactive Explanations. International Journal of Man-Machine Studies, 38, 1993, pp. 169-199.

[CHI 89] CHI M.T.H., BASSOK M., LEWIS M.W., REIMANN P. \& GLASER R. Self-Explanations: How Students Study and Use Examples in Learning to Solve Problems. Cognitive Science, 13, 1989, pp. 145-182.

[CLA 91] CLARK H.H. \& BRENNAN S.E. Grounding in Communication. In L. Resnick, J. Levine and S. Teasley. Perspectives on Socially Shared Cognition [pp. 127-149]. Hyattsville, MD: American Psychological Association, 1991.

[CLA 92] CLANCEY W.J. Representations of Knowing: In Defense of Cognitive Apprenticeship. A response to Sandberg \& Wielinga. Journal of Artificial Intelligence in Education, 3 [2], 1992, pp. 139-168.

[CLA 93] CLANCEY W.J. Guidon-Manage Revisited: A Socio-Technical Systems Approach. Journal of Artificial Intelligence in Education, 4 [1], 1993, pp. 5-34.

[DEL 93] DELOZANNE E. Un projet pluridisciplinaire: ELISE un logiciel pour donner des leçons de méthode. Recherches en Didactique des Mathématiques. Vol. 13, n³, 1993, pp. 1-40.

[DIH 93] DILLENBOURG P., HILARIO M., MENDELSOHN P., SCHNEIDER D. \& BORCIC B. [1993] Intelligent Learning Environments. Final Report of the project 4023 of the National Program on AI \& Robotics [PNR23]. TECFA Document. TECFA, FPSE, Université de Genève, 1993.

[DI 89] DILENBOURG P. Designing a self-improving tutor: PROTO-TEG. Instructional Science, 18, 1989, pp.193-216.

[DIS 92a] DILLENBOURG P. \& SELF J.A. [1992 a] A framework for learner modelling. Interactive Learning Environments. vol. $2, \mathrm{n}^{\circ} 2,1992, \mathrm{pp} .111-$ 137.

[DIS 92b] DILLENBOURG P. \& SELF J.A. A computational approach to socially distributed cognition. European Journal of Psychology of Education., 3 [4], pp. 353-372.

[DIS 93] DLLENBOURG P. \& SCHNEIDER D. Designers did not wait for situationists: A response to Clancey's Viewpoint "Guidon-Manage Revisited" Journal of Artificial Intelligence in Education, 4 [1], 1993, pp. 41-48.

[FRE 88] FREDERIKSEN J.R. \& WHITE B. Intelligent learning systems for science education. Porceedings of ITS-88, Montreal, pp 291-298.

[LAV 88] LAVE J. Cognition in practice. Cambridge: Cambridge University Press, 1988.

[MOY 92] MOYSE R. VIPER: The Design and Implementation of Multiple Viewpoints for Tutoring Systems. in R. Moyse and M. Elsom-Cook (Eds) Knowledge Negotiation. [pp 97-134]. London: Academic Press, 1992.

[NEG 89] NEWMAN D., GRIFFIN P., \& COLE M. The construction zone: working for cognitive change in school. Cambridge University Pres: Cambridge, 1989

[NEW 72] NEWELL A. \& SIMON H.A. Human Problem Solving. New York: Prentice Hall, 1972. 
[NEW 89] NEWMAN D. Is a student model necessary? Apprenticeship as a model for ITS. Proceedings of the 4th AI \& Education Conference [pp.177184], May 24-26 1989. Amsterdam, The Netherlands: IOS.

[OHL 87] OHLSSON, S. Some principles of intelligent tutoring. In R.W. Lawler and M. Yazdani [Eds.], Artificial Intelligence and Education. Vol. I, pp 203-237, 1987. Norwood, NJ: Ablex.

[OMA 87] O'MALLEY Understanding Explanation. Cognitive Science Research Report No CSRP-88, University of Sussex [UK], 1987.

[RES 91] RESNICK L, LEVINE J. \& TEASLEY S (Eds). Perspectives on Socially Shared Cognition [pp. 127-149]. Hyattsville, MD: American Psychological Association, 1991.

[ROG 91] ROGOFF B. Social interaction as apprenticeship in thinking: guided participation in spatial planning. In L. Resnick, J. Levine and S. Teasley. Perspectives on Socially Shared Cognition [pp. 349-364]. Hyattsville, MD: American Psychological Association, 1991.

[ROS 88] ROSCHELLE, J. Sufficiency and Utility of Physics Problem Solving Processes. In Proceedings of the International Conference on Intelligent Tutoring Systems [pp 132-139]. , June 1-3 1988. Montreal, Canada.

[ROS 90] ROSCHELLE, J. Designing for Conversations. Paper presented at the AAAI Symposium on Knowledge-Based Environments for Learning and Teaching, March. 1990 Stanford,CA.

[ROS 92] ROSCHELLE Learning by Collaborating: Convergent Conceptual Change. Journal of the Learning Sciences, 2, 1992, pp. 235-276.

[SAN 92] SANDBERG J. \& WIELINGA B. Situated Cognition: A Paradigm Shift. Journal of Artificial Intelligence in Education, 3 [2], 1992, pp.129-138.

[SEL 88] SELF, J.A. Bypassing the intractable problem of student modelling. Proceedings of the International Conference on Intelligent Tutoring Systems [pp.18-24], June 1-3 1988. Montreal, Canada.

[SHR 90] SCHRAGE M. Shared Minds. New York: Random House, 1990.

[SLE 82] SLEEMAN D. \& BROWN J.S (Eds), Intelligent Tutoring Systems. London: Academic Press, 1982.

[SUC 87] SUCHMAN L.A. Plans and Situated Actions. The problem of humanmachine communication. Cambridge: Cambridge University Press, 1987.

[TWI 89] TWIDALE M.B. Intermediate representations for student errors diagnosis and support. Proceedings of the 4 th $A I \&$ Education Conference [pp. 298-306], May 24-26 1989. Amsterdam, The Netherlands: IOS.

[VIV 88] VIVET M., DELOZANNE E \& CARRIERE E. Presenttaion of differtent aspects of Amalia: A knowledge-Based Tutor for Mathematics. Proceedings of the Summer University on Intelligent Tutoring Systems, Le Mans [France], 1988, pp. 155-164.

[VYG 78] VYGOTSKY L.S. Mind in Society. The Development of Higher Psychological Processes. Edited by M. Cole, V. John-Steiner, S. Scribner \& E. Souberman Harvard University Press. Cambridge, Massachusetts: Harvard University Press, 1978.

[WEN 87] WENGER E. Artificial Intelligence and Tutoring Systems: Computational and Cognitive Approaches to the Communication of Knowledge. Los Altos, CA: Morgan Kaufmann, 1987. 
[WIN 86] WINOGRAD T. \& FLORES F. Understanding Computers and Cognition: A New Foundation for Design. Norwood, NJ: Ablex Publishing Corporation. 